\title{
The Sym-Bobenko Formula and Constant Mean Curvature Surfaces in Minkowski 3-Space
}

\author{
Tetsuya TANIGUCHI \\ Tohoku University \\ (Communicated by T. Nagano)
}

\section{Introduction.}

Recently, Dorfmeister, Pedit and Wu discovered a Weierstrass-type representation for harmonic maps from a Riemann surface into symmetric spaces [DPW]. In their formula, the Weierstrass data are defined as meromorphic potentials, i.e. meromorphic 1-forms on a Riemann surface with values in an infinite-dimensional loop algebra. They regarded a harmonic map as a map taking values in a twisted loop group and showed that every harmonic map from a Riemann surface into a symmetric space is obtained by integrating the potential. In a related paper, Dorfmeister and Haak have constructed constant mean curvature surfaces by applying the Sym-Bobenko formula $[\mathrm{DH}]$ to the loop-group-valued maps given by integrating the potentials.

On the other hand, Kenmotsu discovered a representation formula for immersions with prescribed mean curvature from a simply connected Riemann surface into Euclidean 3-space. In particular, he obtained a formula for an immersion with constant mean curvature whose Gauss map is a given harmonic map [K]. And Akutagawa and Nishikawa constructed the Minkowski 3-space version of the above formula [AN].

Motivated by these results, the present paper has two aims. The first is to establish a natural correspondence between the following two spaces: the space of conformal spacelike immersions with constant mean curvature from a simply connected Riemann surface $\Sigma$ into Minkowski 3-space, and that of nowhere anti-holomorphic harmonic maps from $\Sigma$ into the Poincaré half plane, regarded as the riemannian symmetric space $S L(2, \mathbf{R}) / S O(2)$. The second is to prove the Lorentzian version of the Sym-Bobenko formula and apply it to construct spacelike immersions with constant mean curvature.

In section 2 we shall first prepare notations used in the later sections and recall the identification of the riemannian symmetric space $S L(2, \mathbf{R}) / S O(2)$ with the unit disk equipped with the Poincaré metric and also with the Poincaré half plane. In section 3 we shall define a $\operatorname{sl}(2, \mathbf{R})$-valued 1 -form $\Lambda^{f}$ on a Riemann surface $\Sigma$ associated to a

Received March 18, 1996 
smooth map $f: \Sigma \rightarrow S L(2, \mathbf{R}) / S O(2)$ and show that the harmonicity of $f$ is equivalent to the $d$-closedness of $\Lambda^{f}$. By using this fact, we shall establish the correspondence between the two spaces above-mentioned. In section 4 we shall prove the Sym-Bobenkotype formula and give examples of spacelike immersions with constant mean curvature.

The author thanks Professors S. Nishikawa and S. Nayatani for their advice and encouragement.

\section{Preliminaries.}

We begin with fixing our terminology and notation. Let $L^{3}=\left(\mathbf{R}^{3}, \bar{g}\right)$ denote Minkowski 3-space. Here $\bar{g}$ is the flat Lorentzian metric of signature $(+,+,-)$. In terms of the canonical coordinates $\left(x^{1}, x^{2}, x^{3}\right)$ of $\mathbf{R}^{3}$, the metric $\bar{g}$, denoted also by $\langle$,$\rangle ,$ is expressed as $\bar{g}=\left(d x^{1}\right)^{2}+\left(d x^{2}\right)^{2}-\left(d x^{3}\right)^{2}$. Let $\Sigma$ be a Riemann surface and $\Phi: \Sigma \rightarrow L^{3}$ a smooth map from $\Sigma$ into $L^{3}$. Let $\tilde{\Sigma}$ be the open subset of $\Sigma$ defined by

$$
\tilde{\Sigma}=\left\{p \in \Sigma \mid \Phi^{*} \bar{g} \text { is positive definite at } p\right\} \text {. }
$$

We call $\Phi: \Sigma \rightarrow L^{3}$ a spacelike immersion if $\Sigma=\tilde{\Sigma}$. Throughout this paper, we assume that $\Phi$ is weakly conformal, namely,

$$
\Phi^{*} \bar{g}=\lambda^{2}\left(d \xi^{1} \otimes d \xi^{1}+d \xi^{2} \otimes d \xi^{2}\right), \quad \lambda \geqq 0,
$$

where $\xi=\xi^{1}+\sqrt{-1} \xi^{2}$ is a local complex coordinate on $\Sigma$. Let $I$ be the first fundamental form of the immersion $\left.\Phi\right|_{\mathfrak{F}}$, that is, the riemannian metric on $\tilde{\Sigma}$ obtained by restricting $\Phi^{*} \bar{g}$ to $\tilde{\Sigma}$.

We define a local Lorentzian frame field $\left(e_{1}, e_{2}, e_{3}\right)$ adapted to $\left.\Phi\right|_{\mathfrak{F}}$ as follows. Let $\Phi(\xi)=\left(\Phi^{1}\left(\xi^{1}, \xi^{2}\right), \Phi^{2}\left(\xi^{1}, \xi^{2}\right), \Phi^{3}\left(\xi^{1}, \xi^{2}\right)\right)$ be a local expression of the smooth map $\Phi$ with respect to a local complex coordinate $\xi=\xi^{1}+\sqrt{-1} \xi^{2}$ on $\tilde{\Sigma}$. For $i=1,2$, let

$$
e_{i}=\frac{1}{\lambda} \frac{\partial \Phi}{\partial \xi^{i}}=\frac{1}{\lambda}\left(\frac{\partial \Phi^{1}}{\partial \xi^{i}}, \frac{\partial \Phi^{2}}{\partial \xi^{i}}, \frac{\partial \Phi^{3}}{\partial \xi^{i}}\right) .
$$

We define $e_{3}=e_{1} \times e_{2}$. Here the exterior product $v \times w$ of two vectors $v={ }^{t}\left(x_{1}, x_{2}, x_{3}\right)$, $w={ }^{t}\left(y_{1}, y_{2}, y_{3}\right)$ in $L^{3}$ is defined by

$$
v \times w={ }^{t}\left(x_{3} y_{2}-x_{2} y_{3}, x_{1} y_{3}-x_{3} y_{1}, x_{1} y_{2}-x_{2} y_{1}\right) \quad \text { (cf. [AN]). }
$$

Let II denote the second fundamental form of $\left.\Phi\right|_{\Sigma}$. We denote the covariant differentiation in $L^{3}$ by $D$. If we set

$$
Q=-\left\langle D_{\partial} \partial, e_{3}\right\rangle, \quad H=-\frac{2}{\lambda^{2}}\left\langle D_{\partial} \delta, e_{3}\right\rangle,
$$

$I I$ is expressed as

$$
I I=Q d \xi \otimes d \xi+(1 / 2) H \lambda^{2} d \xi \otimes d \bar{\xi}+(1 / 2) H \lambda^{2} d \bar{\xi} \otimes d \xi+\bar{Q} d \bar{\xi} \otimes d \bar{\xi}
$$


where $\partial=\partial / \partial \xi$ and $\bar{\partial}=\partial / \partial \bar{\xi}$. Notice that $H$ is nothing but the mean curvature of the immersion.

Next we define the Gauss map $G(\Phi): \tilde{\Sigma} \rightarrow \mathscr{H} \subset L^{3}$ of $\left.\Sigma\right|_{\Sigma}$ by $p \mapsto e_{3}(p)$, where $\mathscr{H}$ is the unit pseudosphere defined by $\mathscr{H}=\left\{t(x, y, z) \in L^{3} \mid x^{2}+y^{2}-z^{2}=-1\right\}$.

Next we recall the relation among various models of the hyperbolic plane defined by

$$
\mathbf{H}=\left(\{p+\sqrt{-1} q \in \mathbf{C} \mid q>0\}, \frac{d p \otimes d p+d q \otimes d q}{q^{2}}\right) .
$$

Let $\mathscr{H}^{+}$be the upper unit pseudosphere in $L^{3}$ defined by $\mathscr{H}^{+}=\{t(x, y, z) \in \mathscr{H} \mid z>0\}$. Let $\psi: \mathscr{H}^{+} \rightarrow \mathbf{D}$ be the stereographic projection from $\mathscr{H}^{+}$into the unit disk $\mathbf{D}=\{\alpha \in \mathbf{C}|| \alpha \mid<1\}$ given by

$$
{ }^{t}(x, y, z) \mapsto \frac{x}{1+z}+\sqrt{-1} \frac{y}{1+z} .
$$

Let $\gamma: \mathbf{D} \rightarrow \mathbf{H}$ be the Cayley transform, that is, the map given by

$$
\alpha \mapsto-\sqrt{-1} \frac{\alpha+\sqrt{-1}}{\alpha-\sqrt{-1}},
$$

and $\varphi: \mathscr{H}^{+} \rightarrow \mathbf{H}$ the map defined by $\varphi=\gamma \circ \psi$. Let $J$ be the map from $L^{3}$ to $\operatorname{sl}(2, \mathbf{R})$ defined by

$$
{ }^{t}(x, y, z) \mapsto(x / 2) \eta_{1}+(y / 2) \eta_{2}+(z / 2) \eta_{3},
$$

where

$$
\eta_{1}=\left(\begin{array}{cc}
1 & 0 \\
0 & -1
\end{array}\right), \quad \eta_{2}=\left(\begin{array}{cc}
0 & -1 \\
-1 & 0
\end{array}\right), \quad \eta_{3}=\left(\begin{array}{cc}
0 & -1 \\
1 & 0
\end{array}\right) .
$$

By a simple calculation, we see that $J$ satisfies

$$
J\left(r_{1} \times r_{2}\right)=\left[J\left(r_{1}\right), J\left(r_{2}\right)\right] \quad \text { for any two vectors } r_{1}, r_{2} \text { in } L^{3} \text {, }
$$

where $[$,$] denotes the Lie bracket of \operatorname{sl}(2, \mathbf{R})$ and $\times$ is the exterior product defined as above.

Now we have the natural bijection $\rho: S L(2, \mathbf{R}) / S O(2) \rightarrow \mathbf{H}$ given by

$$
\left(\begin{array}{ll}
a & b \\
c & d
\end{array}\right) S O(2) \mapsto \frac{a \sqrt{-1}+b}{c \sqrt{-1}+d} .
$$

The canonical metric on $S L(2, \mathbf{R}) / S O(2)$ as a riemannian symmetric space coincides with the pull back

$$
\rho^{*}\left(\frac{d p \otimes d p+d q \otimes d q}{q^{2}}\right)
$$


of the Poincaré metric by $\rho$.

PROPOSITION 2.2. For any element $g$ in $S L(2, \mathbf{R})$, we have the following identity

$$
\varphi \circ J^{-1}\left(\operatorname{Ad}(g) \frac{1}{2} \eta_{3}\right)=\rho \circ \pi(g),
$$

where $\pi$ is the natural projection from $S L(2, \mathbf{R})$ to $S L(2, \mathbf{R}) / S O(2)$.

Proof. Setting $g=\left(\begin{array}{ll}a & b \\ c & d\end{array}\right)$, we have

$$
A d(g) \frac{1}{2} \eta_{3}=(a c+b d) \frac{1}{2} \eta_{1}+\frac{1}{2}\left(a^{2}+b^{2}-c^{2}-d^{2}\right) \frac{1}{2} \eta_{2}+\frac{1}{2}\left(a^{2}+b^{2}+c^{2}+d^{2}\right) \frac{1}{2} \eta_{3} .
$$

So $J^{-1}\left(\operatorname{Ad}(g) \frac{1}{2} \eta_{3}\right)$ lies in $\mathscr{H}^{+}$. Mapping this point of $\mathscr{H}^{+}$by $\psi$, we get

$$
\psi \circ J^{-1}\left(A d(g) \frac{1}{2} \eta_{3}\right)=\frac{2(a c+b d)}{2+a^{2}+b^{2}+c^{2}+d^{2}}+\sqrt{-1} \frac{a^{2}+b^{2}-c^{2}-d^{2}}{2+a^{2}+b^{2}+c^{2}+d^{2}} .
$$

By a straightforward computation, $\gamma^{-1} \circ \rho \circ \pi(g)$ is equal to the right-hand side of this formula. This completes the proof of Proposition 2.2.

\section{3. $\operatorname{sl}(2, \mathrm{C})$-valued 1-forms on a Riemann surface.}

Let $\Sigma$ be a Riemann surface and $f: \Sigma \rightarrow S L(2, \mathbf{R}) / S O(2)$ a smooth map. We define an $s l(2, \mathrm{C})$-valued 1-form $\omega^{f}$ on $\Sigma$ as follows. Take any point $p$ of $\Sigma$, and let $(U(p), \xi)$ be a local coordinate system around $p$ so that there exists a local lift $F: U(p) \rightarrow S L(2, \mathbf{R})$. Let $A, B, C$ be complex-valued smooth functions on $U(p)$ such that

$$
A \eta_{1}+B \eta_{2}+C \eta_{3}=F^{-1} \frac{\partial}{\partial \xi} F
$$

Let $\omega^{f}$ be the $s l(2, \mathbf{C})$-valued 1 -form on $\Sigma$ defined by

$$
\left(\omega^{f}\right)_{p}=m(p) A d(F(p)) \sigma_{-} \otimes(d \xi)_{p},
$$

where the complex-valued smooth function $m$ and the element $\sigma_{-}$of $s l(2, \mathrm{C})$ are defined respectively by

$$
m=\sqrt{-1} A-B, \quad \sigma_{-}=\frac{1}{2}\left(\eta_{1}-\sqrt{-1} \eta_{2}\right) .
$$

Let $\omega$ denote the map $f \in C^{\infty}(\Sigma, S L(2, \mathbf{R}) / S O(2)) \mapsto \omega^{f} \in \Gamma\left(\operatorname{sl}(2, \mathbf{C}) \otimes T_{\mathbf{C}}^{* 1,0} \Sigma\right)$.

LEMMA 3.4. The map $\omega$ is well-defined.

Proof. It is easy to check that the definition of $\omega$ is indepednent of the choice of coordinate system. We verify that the definition of $\omega$ is independent of the choice 
of the lift $F$. To do this, let $\widetilde{F}$ be another lift of $f$, i.e. $\tilde{F}=F k$, wehre

$$
k=\left(\begin{array}{cc}
\cos \theta & -\sin \theta \\
\sin \theta & \cos \theta
\end{array}\right)
$$

is a map from $U(p)$ to $S O(2)$. Let $\tilde{A}, \widetilde{B}, \tilde{C}$ and $\tilde{m}$ be the corresponding functions on $U(p)$. Setting $\mu=e^{\sqrt{-1} \theta}$, we get

$$
\tilde{m}=\mu^{2} m, \quad A d(\tilde{F}) \sigma_{-}=\mu^{-2} A d(F) \sigma_{-} .
$$

Thus we see that

$$
\tilde{m} A d(\tilde{F}) \sigma_{-} \otimes d \xi=m A d(F) \sigma_{-} \otimes d \xi .
$$

This completes the proof of Lemma 3.4.

Let $\Lambda^{f}=\omega^{f}+\overrightarrow{\omega^{f}}$, twice the real part of $\omega^{f}$, and $\Lambda$ the map $f \in C^{\infty}(\Sigma, S L(2, \mathbf{R}) /$ $S O(2)) \mapsto \Lambda^{f} \in \Gamma\left(\operatorname{sl}(2, \mathbf{R}) \otimes T_{\mathbf{R}}^{*} \Sigma\right)$.

LEMMA 3.5. Let $f$ be a smooth map from $\Sigma$ to $S L(2, \mathbf{R}) / S O(2)$. Then $f$ is harmonic if and only if $d \Lambda^{f}=0$.

Proof. To start the proof, we quote the following

ThEOREM 3.6 [GO]. Let $G / K$ be a symmetric space and $\pi: G \rightarrow G / K$ the natural projection. We denote the Lie algebras of $G$ and $K$ by $\mathfrak{g}$ and $\mathfrak{f}$ respectively, and let $\mathfrak{g}=\mathfrak{f} \oplus \mathfrak{p}$ be the Cartan decomposition. Let $F: \Sigma \rightarrow G$ be a smooth map from a Riemann surface $\Sigma$ into $G$, and let $\alpha=\alpha_{0}+\alpha_{1}=F^{-1} d F$ where $\alpha_{0}$ and $\alpha_{1}$ are $\mathfrak{1}$ - and $\mathfrak{p}$-valued. Then $\pi \circ F$ is harmonic if and only if

$$
\partial \alpha_{1}^{\prime}+\left[\alpha_{0} \wedge \alpha_{1}^{\prime}\right]=0
$$

where $\alpha_{1}^{\prime}$ is the $(1,0)$-component of $\alpha_{1}$.

We apply this theorem to $G=S L(2, \mathbf{R})$ and $K=S O(2)$. Notice that $\mathfrak{f}=\mathbf{R} \eta_{3}$ and $\mathfrak{p}=\mathbf{R} \eta_{1} \oplus \mathbf{R} \eta_{2}$. Then by substituting (3.1), equation (3.7) becomes the system

$$
\left\{\begin{array}{l}
\frac{\partial}{\partial \bar{\xi}} B=2 \bar{C} A \\
\frac{\partial}{\partial \bar{\xi}} A=-2 \bar{C} B .
\end{array}\right.
$$

By a direct calculation,

$$
\begin{aligned}
d \Lambda^{f}= & -\left(\sqrt{-1} A_{\bar{\xi}}-B_{\bar{\xi}}-2 \sqrt{-1} m \bar{C}\right) A d(F) \sigma_{-} \otimes d \xi \wedge d \bar{\xi} \\
& +\left(-\sqrt{-1} \bar{A}_{\xi}-\bar{B}_{\xi}+2 \sqrt{-1} \bar{m} C\right) A d(F) \sigma_{+} \otimes d \xi \wedge d \bar{\xi}
\end{aligned}
$$

where $\sigma_{+}=\frac{1}{2}\left(\eta_{1}+\sqrt{-1} \eta_{2}\right)$. If $f$ is harmonic, then equation (3.8) holds, and substituting this into (3.9), we get $d \Lambda^{f}=0$, proving the "only if" part of the proposition. To prove 
the "if" part, we consider the Maurer-Cartan equation

$$
d \theta+\frac{1}{2}[\theta \wedge \theta]=0,
$$

where $\theta$ is the left Maurer-Cartan form of $S L(2, \mathbf{R})$. Since $\alpha=F^{-1} d F=F^{*}(\theta)$, we get

$$
d \alpha+\frac{1}{2}[\alpha \wedge \alpha]=0 .
$$

Taking the p-part of this, we see that

$$
d \alpha_{1}+\left[\alpha_{0} \wedge \alpha_{1}\right]=0 .
$$

If we use $A, B$, and $C$, this unravels to become

$$
\left\{\begin{array}{l}
A_{\bar{\xi}}-\bar{A}_{\xi}+2 B \bar{C}-2 \bar{B} C=0, \\
B_{\bar{\xi}}-\bar{B}_{\xi}-2 A \bar{C}+2 \bar{A} C=0 .
\end{array}\right.
$$

On the other hand, since $d \Lambda^{f}=0$, it follows from (3.9) that

$$
\left\{\begin{array}{l}
\sqrt{-1} A_{\bar{\xi}}-B_{\bar{\xi}}-2 \sqrt{-1} m \bar{C}=0, \\
-\sqrt{-1} \bar{A}_{\xi}-\bar{B}_{\xi}+2 \sqrt{-1} \bar{m} C=0 .
\end{array}\right.
$$

Solving equations (3.12) and (3.13), we get (3.8). We have completed the proof of Lemma 3.5.

Let $H$ be a fixed positive constant. For a smooth map $f: \Sigma \rightarrow S L(2, \mathbf{R}) / S O(2)$, we define $L^{3}$-valued 1-form $L^{f}$ on $\Sigma$ by

$$
L^{f}(X)=J^{-1}\left(\frac{1}{H} \Lambda^{f}(X)\right),
$$

where $X$ is an arbitrary tangent vector to $\Sigma$.

LEMMA 3.15. Let $\Sigma$ be a connected, simply connected Riemann surface and $f: \Sigma \rightarrow S L(2, \mathbf{R}) / S O(2)$ a harmonic map. Then there exists a smooth map $\Phi^{f}: \Sigma \rightarrow L^{3}$, unique up to an additive constant, such that

$$
\left(\Phi^{f}\right)^{*}(\Omega)=L^{f},
$$

where $\Omega={ }^{t}(d x, d y, d z)$.

Proof. Since $f$ is harmonic, we have $d \Lambda^{f}=0$ and so $d L^{f}=0$ by Lemma 3.5. Thus we can integrate $L^{f}$ to get a smooth map $\Phi^{f}: \Sigma \rightarrow L^{3}$, determined up to an additive constant, such that $\left(\Phi^{f}\right)^{*}(\Omega)=L^{f}$.

From this point on, we shall always assume that our Riemann surface $\Sigma$ is connected and simply connected. 
LEMMA 3.16. Let $f: \Sigma \rightarrow S L(2, \mathbf{R}) / S O(2)$ be a harmonic map and $\Phi^{f}$ as above. Then the pull back $\left(\Phi^{f}\right)^{*} \bar{g}$ is given by

$$
\Phi^{f *} \bar{g}=\frac{2 m \bar{m}}{H^{2}}(d \xi \otimes d \bar{\xi}+d \bar{\xi} \otimes d \xi)
$$

where $m$ is defined as in (3.3).

Proof. Set $\partial=\partial / \partial \xi, \delta=\partial / \partial \bar{\xi}$. The $d \xi \otimes d \bar{\xi}$ component of $I$ is given by

$$
\left\langle\Phi_{*}^{f} \partial, \Phi_{*}^{f} \bar{\partial}\right\rangle=\left\langle\frac{1}{H} \Lambda^{f}(\partial), \frac{1}{H} \Lambda^{f}(\delta)\right\rangle_{s l}=\frac{2 m \bar{m}}{H^{2}},
$$

where $\langle,\rangle_{s l}$ is the $A d$-invariant inner product of $\operatorname{sl}(2, \mathbf{R})$ given by

$$
\left\langle\eta_{1}, \eta_{1}\right\rangle_{s l}=\left\langle\eta_{2}, \eta_{2}\right\rangle_{s l}=-\left\langle\eta_{3}, \eta_{3}\right\rangle_{s l}=4, \quad\left\langle\eta_{i}, \eta_{j}\right\rangle_{s l}=0 \text { if } i \neq j .
$$

Similary we can compute other components, getting the desired formula.

Proposition 3.17. Let $f: \Sigma \rightarrow S L(2, \mathbf{R}) / S O(2)$ be a smooth map. Take any point $p$ of $\Sigma$, and let $(U(p), \xi)$ be a coordinate neighborhood of $p$ such that $f$ has a local lift

$$
F: U(p) \rightarrow S L(2, \mathbf{R}) ; \xi \mapsto\left(\begin{array}{c}
a(\xi), b(\xi) \\
c(\xi), d(\xi)
\end{array}\right) .
$$

Let $\Psi: \Sigma \rightarrow \mathbf{H}$ be the map defined by

$$
\Psi=\rho \circ f
$$

Then $\Psi_{*}(\partial / \partial \xi)$ is given by

$$
\Psi_{*}\left(\frac{\partial}{\partial \xi}\right)=\left\{m\left(\beta^{1}-\sqrt{-1} \beta^{2}\right)\right\} \frac{\partial}{\partial w}+\left\{(\sqrt{-1} A+B)\left(-\beta^{1}-\sqrt{-1} \beta^{2}\right)\right\} \frac{\partial}{\partial \bar{w}}
$$

on $U(p)$, where $m, A$, and $B$ are defined as in (3.1) and (3.3), $w$ is the complex coordinate of $\mathbf{H}$, and

$$
\beta^{1}=\frac{2\left(d^{2}-c^{2}\right)}{\left(d^{2}+c^{2}\right)^{2}}, \quad \beta^{2}=\frac{4 c d}{\left(d^{2}+c^{2}\right)^{2}} .
$$

In particular, the equation $\partial \Psi / \partial \xi(p)=0$ holds if and only if $m(p)=0$.

Proof. A straightforward computation.

COROLlARY 3.18. Let $f$ and $\Phi^{f}$ be as in Lemma 3.15. Let $\mathscr{N}$ be the subset of $\Sigma$ defined by $\mathcal{N}=\{p \in \Sigma \mid \partial \Psi / \partial \xi(p) \neq 0\}$, where $\Psi=\rho \circ f$. Then $\tilde{\Sigma}=\mathscr{N}$.

Proof. This follows from the definition of $\tilde{\Sigma}$, Lemma 3.16, and Proposition 3.17 .

LEMMA 3.19. Let $f$ and $\Phi^{f}$ be as in Lemma 3.15. Then the image of the Gauss 
map $G\left(\Phi^{f}\right): \tilde{\Sigma} \rightarrow \mathscr{H}$ is contained in $\mathscr{H}^{+}$and

$$
\varphi \circ G\left(\Phi^{f}\right)=\rho \circ f \text { on } \tilde{\Sigma} \text {. }
$$

Proof. Take any point $p \in \tilde{\Sigma}$, and let $(U(p), \xi) \subset \tilde{\Sigma}$ be a coordinate neighborhood of $p$ such that $f$ has a local lift $F: U(p) \rightarrow S L(2, \mathbf{R})$. We can choose

$$
e_{1}=\Phi_{*}^{f}\left(\frac{H}{2 \sqrt{m \bar{m}}} \frac{\partial}{\partial \xi^{1}}\right), \quad e_{2}=\Phi_{*}^{f}\left(\frac{H}{2 \sqrt{m \bar{m}}} \frac{\partial}{\partial \xi^{2}}\right)
$$

as an orthonormal frame on $U(p)$. By definition, the value of the Gauss map $G\left(\Phi^{f}\right)$ at $p$ is given by

$$
\begin{aligned}
G\left(\Phi^{f}\right)(p) & =e_{3}(p)=e_{1}(p) \times e_{2}(p) \\
& =J^{-1}\left(\left[\frac{1}{H} \Lambda^{f}\left(\frac{H}{2 \sqrt{m \bar{m}}} \frac{\partial}{\partial \xi^{1}}\right), \frac{1}{H} \Lambda^{f}\left(\frac{H}{2 \sqrt{m \bar{m}}} \frac{\partial}{\partial \xi^{2}}\right)\right]\right) \\
& =J^{-1}\left(A d(F(p)) \frac{1}{2} \eta_{3}\right) .
\end{aligned}
$$

Using Proposition 2.2, $G\left(\Phi^{f}\right)(p)$ lies in $\mathscr{H}^{+}$and

$$
\begin{aligned}
\varphi \circ G\left(\Phi^{f}\right)(p) & =\varphi \circ\left(J^{-1}\left(A d(F(p)) \frac{1}{2} \eta_{3}\right)\right) \\
& =\rho \circ \pi(F(p))=\rho \circ f(p) .
\end{aligned}
$$

LEMMA 3.20. Let $\Phi^{f}$ be as in Lemma 3.15, and II the second fundamental form of the immersion $\left.\Phi^{f}\right|_{\Sigma}: \tilde{\Sigma} \rightarrow L^{3}$. Then II is given by

$$
I I=\frac{2\left(A^{2}+B^{2}\right)}{H} d \xi \otimes d \xi+\frac{2 m \bar{m}}{H} d \xi \otimes d \bar{\xi}+\frac{2 m \bar{m}}{H} d \bar{\xi} \otimes d \xi+\frac{2 \overline{\left(A^{2}+B^{2}\right)}}{H} d \bar{\xi} \otimes d \bar{\xi},
$$

where $A, B$, and $m$ are defined as in (3.1) and (3.3).

Proof. Set $\Phi=\Phi^{f}$. The $d \xi \otimes d \xi$ component of $I I$ is given by

$$
-\left\langle D_{\partial}\left(\Phi_{*} \partial\right), e_{3}\right\rangle=-\left\langle\frac{1}{H} \partial\left(\Lambda^{f}(\partial)\right), A d(F) \frac{1}{2} \eta_{3}\right\rangle_{s l}=\frac{2\left(A^{2}+B^{2}\right)}{H} .
$$

The other components can be computed in a similar way, and we get the desired formula.

COROLlaRY 3.21. Let $\Phi^{f}$ be as in Lemma 3.15. Then the mean curvature of $\left.\Phi^{f}\right|_{\tilde{\Sigma}}: \tilde{\Sigma} \rightarrow L^{3}$ is equal to the constant $H$.

Próof. Let $I=\left.\Phi^{f *} \bar{g}\right|_{\Sigma}$. The mean curvature of $\left.\Phi^{f}\right|_{\Sigma}$ is given by (1/2)trace $(I I)$. Using Lemma 3.16 and Lemma 3.20, we get $(1 / 2) \operatorname{trace}_{I}(I I)=H$. 
Let Harm denote the space of harmonic maps from $\Sigma$ into $S L(2, \mathbf{R}) / S O(2)$, and let $\widetilde{C}^{\infty}\left(\Sigma, L^{3}\right)$ be the space of equivalence classes of maps from $\Sigma$ into $L^{3}$, where two elements $\Phi_{1}, \Phi_{2}: \Sigma \rightarrow L^{3}$ are equivalent if $\Phi_{2}=\Phi_{1}+c$ for some constant vector $c$ in $L^{3}$. By Lemma 3.15 we have the map

$$
R: \operatorname{Harm} \rightarrow \tilde{C}^{\infty}\left(\Sigma, L^{3}\right) ; \quad f \mapsto\left[\Phi^{f}\right],
$$

where $\Phi^{f}$ satisfies $\Phi^{f *}(\Omega)=L^{f}$. Let Harm* be the set of elements of Harm which are nowhere anti-holomorphic, and denote by $\operatorname{Imm}_{H}\left(\Sigma, L^{3}\right)$ the set of elements of $\widetilde{C}^{\infty}\left(\Sigma, L^{3}\right)$ whose representatives are conformal spacelike immersion with constant mean curvature $H>0$ having Gauss images in $\mathscr{H}^{+}$.

THEOREM 3.22. The image of Harm* by $R$ is contained in $\operatorname{Imm}_{H}\left(\Sigma, L^{3}\right)$. Moreover $R^{*}: \operatorname{Harm}^{*} \rightarrow \operatorname{Imm}_{H}\left(\Sigma, L^{3}\right)$ is bijective, where $R^{*}$ is the restriction of $R$ to Harm*.

Proof. The first statement follows immediately from Lemma 3.16, Corollary 3.18, Lemma 3.19 and Corollary 3.21. Let us prove the bijectivity of $R^{*}$. Since the Gauss map of an immersion with constant mean curvature is harmonic [M], we can define the map $\tilde{G}: \operatorname{Imm}_{H}\left(\Sigma, L^{3}\right) \rightarrow$ Harm by $[\Phi] \mapsto \rho^{-1} \circ \varphi \circ G(\Phi)$. First we shall show that the image of $\tilde{G}$ is contained in Harm*. Assume that $\tilde{G}([\Phi])$ is not a nowhere anti-holomorphic map, i.e. $\Psi=\psi \circ G(\Phi)$ has some point $p \in \Sigma$ such that $\partial \Psi / \partial \xi(p)=0$. Since the induced metric $\Phi^{*} \bar{g}$ is given by

$$
\Phi^{*} \bar{g}=\left[\frac{1}{H} \frac{2}{\left(1-|\Psi|^{2}\right)}\left|\frac{\partial \Psi}{\partial \xi}\right|\right]^{2}\left(\left(d \xi^{1}\right)^{2}+\left(d \xi^{2}\right)^{2}\right)
$$

(See $[\mathrm{AN}].), \Phi$ is degenerate at the point $p$. (Note that the orientation of $\mathbf{D}$ in this paper is opposite to the one in [AN]. So the above expression of the metric is slightly different from that in [AN].) This contradicts the assumption that $\Phi$ is an immersion. So $\tilde{G}$ maps $\operatorname{Imm}_{H}\left(\Sigma, L^{3}\right)$ into Harm*. We have $\tilde{G} \circ R^{*}=\mathrm{id}$, as is easily derived from Lemma 3.19. So to prove the bijectivity of $R^{*}$, it remains to show that $\tilde{G}$ is injective. Let $\left[\Phi_{1}\right],\left[\Phi_{2}\right]$ be two elements of $\operatorname{Imm}_{H}\left(\Sigma, L^{3}\right)$ such that $\tilde{G}\left(\left[\Phi_{1}\right]\right)=$ $\tilde{G}\left(\left[\Phi_{2}\right]\right)$. Since the Gauss maps and the mean curvatures of $\Phi_{1}$ and $\Phi_{2}$ agree, their first and the second fundamental forms must also agree. By the fundamental theorem of differential geometry, there exists a rotational isometry $\sigma$ of $L^{3}$ and a constant vector $c$ of $L^{3}$ such that $\Phi_{2}=\sigma \circ\left(\Phi_{1}+c\right)$ and $\sigma(0)=0$. Combining this and the assumption that $G\left(\Phi_{1}\right)=G\left(\Phi_{2}\right)$, we have $G\left(\Phi_{1}\right)=\sigma\left(G\left(\Phi_{1}\right)\right)$. Suppose that $\sigma$ is not the identity. Then since $\sigma$ is an orientation preserving rotational isometry of $L^{3}$, which fixes $0 \in L^{3}$ and satisfies $\sigma\left(\mathscr{H}^{+}\right)=\mathscr{H}^{+}, \sigma$ has at most one fixed point in $\mathscr{H}^{+}$. So $\tilde{G}\left(\left[\Phi_{1}\right]\right)$ is a constant map and, in particular, is an anti-holomorphic map. This contradiction shows that $\sigma$ must be the identity, and so $\Phi_{1}=\Phi_{2}+c$, i.e. $\left[\Phi_{1}\right]=\left[\Phi_{2}\right]$. Thus $\tilde{G}$ is injective. Theorem 3.22 has been proved. 


\section{The Sym-Bobenko formula for Minkowski 3-space.}

We shall derive the Sym-Bobenko formula for Minkowski 3-space.

THEOREM 4.1. Let $\Sigma$ be a simply connected Riemann surface and $f: \Sigma \rightarrow S L(2, \mathbf{R}) /$ $S O(2)$ a hormonic map. Let $F: \Sigma \rightarrow S L(2, \mathbf{R})$ be a lift of $f$. Let $F(\cdot):(-\varepsilon, \varepsilon) \times \Sigma \rightarrow$ $S L(2, \mathbf{R}), \varepsilon>0$, be a smooth map such that

(1) $F(0)=F$,

(2)

$$
\left.\frac{d}{d t}\right|_{t=0}\left(F(t)^{-1} \frac{\partial}{\partial \xi} F(t)\right)=-\sqrt{-1}\left(A \eta_{1}+B \eta_{2}\right),
$$

where $A \eta_{1}+B \eta_{2}+C \eta_{3}=F^{-1} \frac{\partial}{\partial \xi} F$. Then $R(f)$ is given by

$$
R(f)=\left[J^{-1}\left\{-\frac{1}{2 H}\left(\left(\left.\frac{d}{d t}\right|_{t=0} F(t)\right) F^{-1}-A d(F) \frac{1}{2} \eta_{3}\right)\right\}\right] .
$$

Proof. Set $[\Phi]=R(f)$. It suffices to show that

$$
\frac{\partial \Phi}{\partial \xi}=\frac{\partial}{\partial \xi}\left\{J^{-1}\left\{-\frac{1}{2 H}\left(\left(\left.\frac{d}{d t}\right|_{t=0} F(t)\right) F^{-1}-A d(F) \frac{1}{2} \eta_{3}\right)\right\}\right\}
$$

Equation (4.2) is equivalent to

$$
J\left(\frac{\partial \Phi}{\partial \xi}\right)=\frac{\partial}{\partial \xi}\left\{-\frac{1}{2 H}\left(\left(\left.\frac{d}{d t}\right|_{t=0} F(t)\right) F^{-1}-A d(F) \frac{1}{2} \eta_{3}\right)\right\} .
$$

By the definition of $R(f)$ and the property of $L^{f}$, the left-hand side of (4.3) is equal to

$$
\frac{1}{H} \Lambda^{f}(\partial)=\frac{1}{H} m A d(F) \sigma_{-} .
$$

On the other hand, $-2 H$ times the right-hand side of (4.3) is equal to

$$
\begin{aligned}
& \frac{\partial}{\partial \xi}\left(\left(\left.\frac{d}{d t}\right|_{t=0} F(t)\right) F^{-1}\right)-\frac{\partial}{\partial \xi}\left(A d(F) \frac{1}{2} \eta_{3}\right) \\
& \quad=\left(\partial\left(\left.\frac{d}{d t}\right|_{t=0} F(t)\right)\right) F^{-1}+\left.\frac{d}{d t}\right|_{t=0} F(t) \partial\left(F^{-1}\right)-A d(F)\left[F^{-1} \partial F, \frac{1}{2} \eta_{3}\right] \\
& =A d(F)\left\{\left.\frac{d}{d t}\right|_{t=0}\left(F(t)^{-1} \partial F(t)\right)\right\}-A d(F)\left(A \eta_{2}-B \eta_{1}\right) \\
& =A d(F)\left\{-\sqrt{-1}\left(A \eta_{1}+B \eta_{2}\right)-\left(A \eta_{2}-B \eta_{1}\right)\right\}=-2 m A d(F) \sigma_{-} .
\end{aligned}
$$

Combining (4.4) and (4.5), we get (4.3). 
EXAMPle 4.6. Let $f: \mathbf{C} \rightarrow \mathbf{H}$ be the harmonic map defined by

$$
\xi=x+\sqrt{-1} y \mapsto \sqrt{-1} \exp (4 y),
$$

and let $f: \mathbf{C} \rightarrow S L(2, \boldsymbol{R}) / S O(2)$ be the harmonic map defined by $f=\rho^{-1} \circ \widetilde{f}$. Let $F(\cdot):(-\pi, \pi) \times \mathbf{C} \rightarrow S L(2, \mathbf{R})$ be the map defined by

$$
(t, x+\sqrt{-1} y) \mapsto\left(\begin{array}{cc}
\exp \left(-\sqrt{-1} \lambda^{-1} \xi+\sqrt{-1} \lambda \bar{\xi}\right) & 0 \\
0 & \exp \left(\sqrt{-1} \lambda^{-1} \xi-\sqrt{-1} \lambda \bar{\xi}\right)
\end{array}\right),
$$

where $\lambda=\exp (\sqrt{-1} t)$. Set $H=1$. Then $F(\cdot)$ satisfies the conditions of Theorem 4.1, and $R(f)$ is given by

$$
R(f)=\left[x+\sqrt{-1} y \mapsto\left(2 x, \frac{\sinh 4 y}{2}, \frac{\cosh 4 y}{2}\right)\right] .
$$

This is a hyperbolic cylinder of mean curvature 1 in $L^{3}$.

EXAMPLe 4.7. Let $f: \mathbf{H} \rightarrow \mathbf{H}$ be the identity map, and let $f=\rho^{-1} \circ \tilde{f}: \mathbf{H} \rightarrow$ $S L(2, \mathbf{R}) / S O(2)$. Let $F(\cdot):(-\pi, \pi) \times \mathbf{H} \rightarrow S L(2, \mathbf{R})$ be the map defined by

$$
(t, x+\sqrt{-1} y) \mapsto\left(\begin{array}{cc}
\sqrt{y} & x / \sqrt{y} \\
0 & 1 / \sqrt{y}
\end{array}\right)\left(\begin{array}{cc}
\cos (1 / 2) t & \sin (1 / 2) t \\
-\sin (1 / 2) t & \cos (1 / 2) t
\end{array}\right) .
$$

Set $H=1$. Then $F(\cdot)$ satisfies the conditions of Theorem 4.1 , and $R(f)$ is given by

$$
R(f)=\left[x+\sqrt{-1} y \mapsto\left(\frac{x}{y},-\frac{1}{2 y}+\frac{y}{2}+\frac{x^{2}}{2 y}, \frac{1}{2 y}+\frac{y}{2}+\frac{x^{2}}{2 y}\right)\right] .
$$

This is a hyperboloid of mean curvature 1 in $L^{3}$.

\section{References}

[AN] K. Akutagawa and S. Nishikawa, The Gauss map and spacelike surfaces with prescribed mean curvature in Minkowski 3-space, Tôhoku Math. J. 42 (1990), 67-82.

[DPW] J. Dorfmeister, F. PediT and H. WU, Weierstrass type representation of harmonic maps into symmetric spaces, preprint.

[DH] J. DorfmeIsTER and G. HAAK, Meromorphic potentials and smooth CMC surfaces, preprint.

[GO] M. A. GUEST and Y. OHNITA, Actions of loop groups, deformations for harmonic maps and their applications, Sugaku 46 (1994), 228-242 (in Japanese).

[K] K. KENMOTSU, Weierstrass formula for surfaces of prescribed mean curvature, Math. Ann. 245 (1979), 89-99.

[M] T. K. MiLnoR, Harmonic maps and classical surface theory in Minkowski 3-space, Trans. Amer. Math. Soc. 280 (1983), 161-185.

\section{Present Address:}

Mathematical Institute, Tohoku University, SENDAI, 980 JAPAN.

e-mail: tetsu@math.tohoku.ac.jp 